\title{
CONSENSO PARA EL DIAGNÓSTICO Y MANEJO DE LA OSTEOPOROSIS
}

Jurado: Fernande Chalem, MD.. Jaime Casashuenas, MD., Hernando Gaitain. MD., José Fernando Grimez. MD., Gilherto) Martínez, MD., Martha Torres de Restrepo. Martha Castaño de Grimes. Martha Castillo de Baracaldo. Mariera Jaramillo. Marisol Ortega. Maria Carolina Niño. MD., Mauricion Bethes. MD.. Leonor Lana.

\section{Segunda parte}

\section{Ejercicio y rehabilitación}

\section{El ejercicio físico como prevención de la osteoporosis}

El ejercicio físico $(\mathrm{EF})$ desde tiempo atrás es considerado como una de las medidas preventivas más importantes en el tratamiento de la osteoporosis, pues junto al tratamiento farmacológico y a una dieta balanceada, aportaelementos que coadyuvan tanto al mantenimiento como al incremento de la calidad del hueso.

Durante la infancia y la adolescencia, se forma cerca del $90 \%$ de la masá ósea, razón por la cual es necesario promover el ejercicio físico y establecer políticas que desde el ámbito educativo, promuevan que incluyan la actividad física regular, enfatizando en ejercicio de cargal de peso.

El papel preventivo del ejercicio físico está determinado no sólo por el efecto que tiene sobre el proceso de remodelado óseo, sino también porque puede reducir el riesgo de caídas que podrían terminar en fiacturas.

Es necesario incluir el EF como factor fundamental en la prevención de la osteoporosis sin que sea una labor dejada para los adultos mayores, sino al contrario iniciarla desde la primera infancia, etapa en la cual los pediatras y médicos de familia, tienen un papel relevante.

Reconociendo que las fracturas son la mayor y más dramática expresión de la osteoporosis, el EF juega un papel fundamental en la prevención de las caídas, puesto que genera en el individuo mayor capacidad de respuesta, manteniendo un equilibrio adecuado entre los grupos musculares.

\section{Efecto beneficio del ejercicio sobre la masa y calidad óseas}

La extrema inactividad y la máxima actividad producen efectos en la masá ósea. La positiva relación entre EF y DMO ha sido documentada en estudios de corte transversal; adicionalmente, la contracción muscular ha sido determinada como un buen predictor de DMO en fémur, columna y antebrazo en mujeres de 20 a 73 años de edad.

El entrenamiento mecánico óptimo para demostrar el efecto de remodelado óseo realizado en animales de experimentación mostró cambios en masa ósea más como resultado de la magnitud de la carga que debidos al ciclo de carga, loque sugiere que la fatiga muscular sea una importante precondición o estímulo para alcanzar remodelado óseo.

Igualmente en trabajo de experimentación encontraron que se requiere de un pequeño número de ciclos de carga para incrementar DMO, aspectos que se han tenido en cuenta para el diseño de programas de EF en humanos

Los ejercicios dinámicos que aplican cargas altas de magnitud y frecuencia, así como aquellos desarrollados durante el entrenamiento de resistencia, pueden cargar efectivamente el esqueleto.

\section{Programa de ejercicio para el paciente con osteoporosis}

Aunque se ha demostrado que el EF de carga de peso influencia el metabolismo óseo, es más controvertido qué tipo de actividad beneficia el hueso. 


\section{Ejercicio aeróbico}

El efecto del ejercicio aeróbico sobre el tejido óseo con actividades tales como bailar, caminar y trotar mantienen el CMO en el radio y cúbito, tanto en mujeres pre como en mujeres posmenopáusicas. Bravo y colaboradores, 1996, han demostrado que la actividad aeróbica preserva la DMO en la columna de mujeres osteopénicas, mientras que hubo descenso significativo en el grupo control.

Heinonen y cols, 1998, investigaron los efectos del entrenamiento de resistencia durante 18 meses (caminata, trote, bicicleta estática, escaladores) al 55 a $75 \%$ de la capacidad aeróbica sobre la DMO en mujeres posmenopáusicas, encontrando un incremento de la DMO a nivel del cuello femoral, en tanto que en el grupo control y en el de actividad calisténica se observó disminución a este nivel.

Caminar exclusivamente, aunque pareciera ser el ejercicio ideal, puede no ser una actividad suficiente para estimular cambios en la DMO. Cuando caminar sea doloroso o cause dificultad, como en algunas lesiones articulares tipo artritis reumatoide $o$ artrosis degenerativas, el uso de bicicleta estática es una opción adicional, pues a la vez que mejora capacidad cardiopulmonar, estimula la formación ósea en columna lumbar.

\section{Ejercicio de carga o de resistencia}

Un protocolo de $\mathrm{EF}$ que produce alto pico de fuerza y tensión, que incluye unas cuantas repeticiones y que sobrecargan todo el hueso, pueden ser más efectivos para mantener y construir DMO: la clásica descripción parece ser más de entrenamiento de carga que de entrenamiento aeróbico, entendida ésta como la resistencia a que se ve sometido el organismo cuando se trabaja contra gravedad por ejemplo trotando o caminando, donde tal resistencia es ofrecida por el piso al contacto con el cuerpo o cuando hay que vencer la resistencia que opone el levantar pesas. Un protocolo de entrenamiento progresivo que incrementa la fuerza muscular puede mejorar la DMO, por vía de la contracción muscular.

Estudio control en grupos de mujeres posmenopáusicas sobre magnitud de carga (intensidad) o número de ciclos de carga(repetición) encontró que ambos grupos ganaron en fuerza, la DMO sólo mejoró en el programa de alta carga y baja repetición. Tales resultados sugieren que la carga máxima es un factor más importante que el número de ciclos de repetición para efectos de mejorar la DMO.

Las pocas investigaciones que estudian la relación dosis respuesta sugieren que el entrenamiento de fuerzas de alta intensidad, que involucre altas cargas con pocas repeticiones, resultan en una mejor DMO.

\section{Prescripción del ejercicio físico}

En este sentido, el EF siempre deberá considerarse cual si fuera un medicamento, esto es, tiene indicaciones, contraindicaciones y precauciones. Por ello, quien lo prescribe, debe conocer los beneficios y riesgos que el EF implica para cada uno de sus pacientes en forma individualizada.

Se sugiere que las mujeres con osteoporosis deben evitar el EF que: 1) cause vibración en la espalda, como en aquellos deportes de alto impacto (trote intenso, aeróbicos de alto impacto); 2) involucre flexión del tronco; 3) incremente el riesgo de caída (patinaje, trampolines, etc.) o 4) implique movimientos de abducción aducción de cadera. Al contrario, formas adecuadas de EF incluyen caminar, fortalecimiento muscular y ejercicios de extensión de columna, siempre y cuando su realización no provoque dolor.

Los ejercicios de carga de peso pueden ser útiles en los programas de prevención de OP. Basados en hallazgos investigativos, los ejercicios que producen altas fuerzas de resistencia como el trote y los escaladores, provocan un mejoramiento significativo en la DMO. El ejercicio de carga provee una eficiente vía para estimular sitios clínicamente importantes como columna, cadera y antebrazo y también tiene beneficios extraesqueléticos por el desarrollo de mayor fuerza muscular y de balance y equilibrio para reducir el riesgo de fractura por caídas.

De otra parte, programas de ejercicio que incluyan el consumo de calcio para favorecer una mayor formación ósea, producen un efecto positivo sobre la DMO, 
efecto que se potencializa, si se adiciona TRH. No existe mucha evidencia del efecto sumatorio del EF frente a la vitamina $\mathrm{D}$, los bisfosfonatos y la calcitonina, aspecto que podría ser de utilidad analizar. Tampoco existe evidencia que permita sugerir el uso de corriente eléctrica en el tratamiento y/o prevención de la osteoporosis.

Sobre estos conceptos se podría concluir que el EF ideal para estimular la DMO debe incluir entrenamiento de resistencia progresiva usando pocas repeticiones al 70 - $80 \%$ de una repetición máxima hasta que los músculos se hayan fatigado.

El EF debe realizarse al menos 3 veces/semana durante 30 minutos y continuarse por lo menos durante un año. Estudios como el de Fries, 1996, muestran que hay reversión de las ganancias en la masa ósea cuando se descontinúa el EF.

La intensidad podrá irse ajustando mensualmente y el ejercicio deberá incluir preferiblemente actividades que involucren grandes grupos musculares en extremidades superiores e inferiores y tronco. Elemento adicional, es incorporar el condicionamiento cardiorrespiratorio, preparador para la actividad.

Si el ejercicio produce molestias o dolor debe interrumpirse, consultar a un experto para analizar si hay algún factor no controlado en su ejecución y modificarlo o definitivamente suspenderlo.

En pacientes de edad avanzada se deben preferir los ejercicios de fortalecimiento, de corrección de postura y de resistencia. Se pueden aplicar a ellos ejercicios isotónicos, aeróbicos, rítmicos dentro de patrones progresivos de tolerancia.

Los pacientes con historia de fractura vertebral por compresión, deben evitar actividades de flexoextensión lumbar, pues se incrementa el riesgo de futuras fracturas.

\section{Nutrición como factor preventivo de osteoporosis}

La mineralización ósea se inicia desde la vida intrauterina y continúa un activo proceso de remodelación a lo largo de toda la vida. El pico máximo de masa ósea se obtiene durante la segunda década de la vida.

Después de la cuarta década se observa un progresivo aumento de pérdida ósea. Por ejemplo, en la mujer premenopáusica se calcula que la pérdida ósea vertebral va de $1 \%$ a $1,6 \%$ por año.

Esto muestra la relación entre pérdida ósea y envejecimiento, y por lo tanto, el riesgo de presentar osteoporosis a medida que pasan los años. Es por ello que se ha popularizado una comparación y una invitación a la vez: ahorre calcio para la vejez. Es como un banco: si tiene suficientes reservas puede gastar y solventar sus necesidades sin quedar en saldo rojo. Pero, si no las hay, se verá en problemas.

No se puede ahorrar cuando ya se es viejo, hay que comenzar desde la niñez y aquí juega papel importante la educación familiar, el apoyo de maestros, padres, pediatras para crear una cultura alimentaria adecuada.

Entonces, el consumo adecuado de calcio desde la niñez es determinante en la formación ósea y representa un aporte fundamental en la prevención de la osteoporosis. Si en la juventud y la adultez temprana se alcanza un pico máximo de densidad ósea, habrá un menor riesgo de osteoporosis.

La mayoría de la población es omnívora y requiere de este comportamiento para alcanzar los requerimientos nutricionales de macro y micronutrientes. Además, la alimentación del hombre se realiza en forma de comidas mixtas que incluyen diversos grupos de alimentos vegetales y animales, y con preparaciones y presentaciones variables, lo que significa que siempre existe una interacción entre los alimentos que se consumen y la absorción o disponibilidad real en el tubo digestivo de cada uno de los nutrimentos.

El secreto está en la ingesta de un porcentaje adecuado de calcio, y en esto es importante tener en cuenta parámetros mundiales. Al no tener una tabla específica para Colombia transcribimos la norteamericana, diseñada en 1994, que señala un consumo promedio, así: 


\begin{tabular}{llll}
\hline Lactantes & $\begin{array}{l}0 \text { a } 6 \text { meses } \\
6 \text { meses a I año }\end{array}$ & $\begin{array}{l}400 \mathrm{mg} / \text { dia } \\
600 \mathrm{mg} / \text { dia }\end{array}$ \\
\hline Nifíos & $\begin{array}{l}\text { I a } 5 \text { años } \\
6 \text { a } 10 \text { años }\end{array}$ & $\begin{array}{l}800 \mathrm{mg} / \text { día } \\
1.200-1.500 \mathrm{mg} / \text { día }\end{array}$ \\
\hline $\begin{array}{l}\text { Adolescentes } \\
\text { y adultos } \\
\text { jóvenes }\end{array}$ & 11 a 24 años & $1.200-1.500 \mathrm{mg} /$ día \\
\hline Hombres & $\begin{array}{l}25-65 \text { años } \\
\text { Más de } 65 \text { años }\end{array}$ & $1.000 \mathrm{mg} /$ día \\
\hline Mujeres & $\begin{array}{l}25 \text { a } 50 \text { años } \\
\text { Más de } 50 \text { años }\end{array}$ & $1.000 \mathrm{mg} /$ día \\
\hline $\begin{array}{l}\text { Sin terapia } \\
\text { estrogénica } \\
\text { Embarazo } \\
\text { lactancia }\end{array}$ & $1.500 \mathrm{mg} /$ día \\
\hline
\end{tabular}

Los requerimientos de calcio varían a lo largo de la vida. Son mayores las necesidades durante los primeros años, en la pubertad, el embarazo, la lactancia y a partir de la premenopausia. En estos momentos se requiere un poco más. Así, se estima que en las mujeres premenopáusicas un aumento de calcio en la dieta, entre 500 y $600 \mathrm{mg} /$ día.

Los alimentos de origen animal tienen un contenido de calcio superior al de los alimentos de origen vegetal, correspondiendo a la leche y todos sus derivados, figura en el primer lugar como la principal fuente de calcio, en la mayoría de las culturas.

\section{La pregunta es \\ ¿en donde está?}

Según la tabla de composición de alimentos del Instituto Colombiano de Bienestar Familiar, estas son algunas fuentes dietarias de calcio:

\begin{tabular}{ll}
\hline \multicolumn{2}{l}{ Origen animal } \\
\hline I vaso de leche $(200 \mathrm{cc})$ & $240 \mathrm{mg}$ \\
\hline I onza de queso & $240 \mathrm{mg}$ \\
\hline I vaso de yogurt & $222 \mathrm{mg}$ \\
\hline I vaso de kumis & $212 \mathrm{mg}$ \\
\hline I onza cuajada & $147 \mathrm{mg}$ \\
\hline I huevo & $27 \mathrm{mg}$ \\
\hline 2 onzas de carne de res magra & $3,6 \mathrm{mg}$
\end{tabular}

\begin{tabular}{lc}
\multicolumn{2}{c}{ Origen vegetal } \\
\hline I porción de repollo $(25 \mathrm{~g})$ & $103 \mathrm{mg}$ \\
\hline I onza de fríjol blanco & $72,9 \mathrm{mg}$ \\
\hline I onza de soya & $63 \mathrm{mg}$ \\
\hline I porción de espinaca $(25 \mathrm{~g})$ & $29,5 \mathrm{mg}$ \\
\hline I pedazo de papaya $(100 \mathrm{~g})$ & $25 \mathrm{mg}$ \\
\hline I astilla de yuca $(50 \mathrm{~g})$ & $14 \mathrm{mg}$ \\
\hline I papa con cáscara $(100 \mathrm{~g})$ & $4 \mathrm{mg}$ \\
\hline I onza de arroz & $2,7 \mathrm{mg}$
\end{tabular}

\section{Absorción y retención}

No todo el calcio que se consume va a los huesos. Existen unas pérdidas obligadas de calcio en el humano que oscilan entre 200 y $300 \mathrm{mg}$ diarios y que ocurren a través de la piel, del aparato gastrointestinal y de los riñones. La mayor cantidad de calcio que se ingiere es absorbida en el intestino delgado y en su proceso intervienen factores como el $\mathrm{pH}$ gástrico, la composición de las comidas, lo cual va directamente a afectar su biodisponibilidad.

Así, un factor a tener en cuenta es la disponibilidad del calcio de los diferentes alimentos, es decir, la posibilidad de absorberse. En este punto, los alimentos de origen animal vuelven a tener la primacía, debido a que la proporción de calcio absorbible es mayor en estos y el calcio absorbido final es superior para un contenido equivalente entre dos porciones de alimento animal y vegetal.

El contenido de calcio en los alimentos y su posibilidad de absorberse son determinantes principales de la absorción del calcio procedente de la alimentación y secundariamente esta obedece a otros múltiples factores como contenido de proteínas, aminoácidos azufrados, lisina, fósforo, sodio, potasio, magnesio, fitatos, oxalatos, fibra vegetal, azufre, ácidos, álcalis y su balance final, cafeína, fitoesteroles y flavonoides, entre otros. A esto se agrega el genotipo individual que determina una mayor o menor proporción de absorción del calcio para una carga específica y que determina el amplio coeficiente de variación de biodisponibilidad del calcio alimentario en un grupo o población. 
La suma de todos estos factores determina el balance final del calcio en el ser humano, de los cuales unos son favorables y otros desfavorables. A los primeros pertenecen el contenido de calcio del al imento, la cantidad ingerida de cada uno, el aporte proteinico total y la calidad de las proteínas acordes con los requerimientos del individuo, fósforo, potasio, magnesio y aporte total de álcalis, fitoesteroles y flavonoides.

Los alimentos de origen animal se caracterizan por tener un elevado contenido proteínico y de alta calidad, lo que favorece y permite un adecuado crecimiento y desarrollo, no solo del tejido magro sino del escyuelético con el logro de la estatura y estructura óseal genéticamente programadals.

El mantenimiento en la edad adulta de la masa ósea y magra depende también de un aporte proteico adecuado y en caso de pérdida favorecen la recuperación y evitan un mayor deterioro en edades en las cuales el balancecálcicoes inevitablemente negativo.

El fóstoro de la alimentación favorece la reabsorción tubular renal de calcio, en cambio, el procedente de las bebidas carbonaladas, con un elevado contenido de ácidos fosfóricos y cítrico, conducen a acidosis y pérdida consecuente de calcio en la orina. Aeste mecanismo se oponen el potasio y el magnesio, los cuales son los determinantes de la alcalinidad de la alimentación y entonces nos referimos al balance global entre ácidos y álcalis al imentarios. Los estudios han demostrado cómo, un balance dietario positivo de ácidos conduce a acidemia crónica y favorece la pérdida de calcio óseo, para neutralizar dicho exceso, el cual es eliminado por vía urinaria. El balance a favor de los álcalis es protector del calcio óseo, favoreciendo su depósito y reduciendo su reabsorción y excreción renal.

En cuanto a los factores dietarios desfavorables para el metabolismo y balance cálcicos, además de los ya mencionados (desnutrición proteico calórica, aporte excesivo de ácidos, aporte cálcico insuliciente), el exceso de sodio juega un papel importante, dado yue la eliminación a través de los riñones de dicho exceso conlleva eliminación de calcio. Por eso, la recomendación es no abusar de la sal. En este punto tendría una ligera ventaja la dieta vegetariana, baja en sal.
El elevado contenido de fïtatos, oxalatos y fïbra. característicos de los alimentos vegetales tiende a formar compuestos insolubles con el calcio dietario y así disminuye la lasa de absorción, en contraposición a los alimentos de origen vegetal, con bajo contenido delos mismos.

Así, una diela normal está dada por la presencia de ambos tipos de alimentos, y el balance timal, yue es la sumatoria de todos y cada uno, permite tener un índice adecuado de calcio, para asegurar una massa ósea adecuada y prevenir la osteoporosis.

\section{Cafeína y metabolismo del calcio}

El consumo de cafeina se ha asociado con disminución de la masá ósea y el consiguiente incremento en el riesgo de fractura. No se dice la última palabra. Es más. hay estudios que plantean diferentes puntos de vista: algunos autores basiados en la influencia de la dieta sobre la masa ósea no encontraron una relación significativa entre el consumo de cafeína y la pérdida de masa ósea. De la misma forma, existen otros estudios donde se encuentra una relación positiva entre el consumo de cafeína y el riesgo de fractura.

Desde el punto de vista lisiopatológico se considera que la caféina aumenta la excreción urinaria de calcio. Para cue esta situación sea suficientemente importante y llegue a manifestarse como disminución en la densidad mineral ósea con incremento de riesgo de fractura, se recuiere un consumo excesivo de café, es decir, más de 5 tazas al día. En conclusión, mientras la ingesta de esta bebida no sea excesival. no debe considerarse como factor de riesgo.

\section{La importancia de la vitamina D}

La Vitamina D es importante para la rijación del calcio por parte del organismo. La principal fuente es el sol, que se debe tomar con protección para prevenir otros problemats como el cáncer de piel. También algunos alimentos contienen vitamina D. La exposición requiere ciertas medidas de prevención, pues el abuso 
podría exponer a otro serio problema como es el cáncer de piel. Se sugiere exponer especialmente la espalda, sitio considerado ideal para la absorción de la vitamina D, estar con cantidades de ropa mínimas, preferiblemente en las primeras horas de la mañana $(8 \mathrm{a}$ 10 de la mañana). El tiempo de exposición no debe exceder de 15 a 20 minutos día, y puede hacerse a través de cristales o directamente, siempre y cuando se utilicen medidas de protección como bloqueadores solares, vísceras o sombreros, así como gafas.

En algunos casos están indicados suplementos de vitamina D, pero no en personas jóvenes. Siempre debe darse bajo supervisión médica, con un monitoreo permanente, y en la mayoría de los casos se utilizan como un complemento de la terapia de manejo de la osteoporosis.

Igualmente merecen atención personas con deficiencias hepáticas, cuando es indispensable en ellos la administración de vitamina D. En los ancianos es necesario administrar este suplemento, teniendo en cuenta la farmacocinética y farmacodinámica de su organismo. También lo necesitan los pacientes con raquitismo, osteoporosis, insuficiencia respiratoria crónica, y pacientes con terapias de glucocorticoides en forma prolongada.

En general, la utilización de la vitamina D en el tratamiento de la osteoporosis está justificada por la deficiencia que se ha documentado, especialmente en la osteoporosis tipo II y en la tipo I de acuerdo a los estudios en mujeres postmenopáusicas con baja exposición al sol. No se ha demostrado que los suplementos sean efectivos en la prevención de la osteoporosis postmenopáusica.

\section{Suplementos, ¿sí o no?}

Aunque los requerimientos de calcio se pueden cubrir con una buena alimentación, existen una serie de suplementos que bien indicados resultan favorables. Es más, se estima que los suplementos de calcio son útiles en todas las edades y son parte obligada de los esquemas terapéuticos para osteoporosis en prevención y tratamiento. Si les adicionan vitamina $\mathrm{D}$, permiten una mejor absorción intestinal, lo que es más recomendable para pacientes con pobre exposición al sol y que reciben glucocorticoides y que padecen IRC.
Recientes estudios demuestran que los suplementos de calcio y vitamina $D$ en pacientes ancianos, mayores de 75 años, tienen una equivalencia similar en cuanto a beneficios en pacientes con osteoporosis y riesgo de fractura.

En mujeres postmenopáusicas, por ejemplo, se sabe que la pérdida de masa ósea se previene realizando suplementación combinada de calcio y dosis bajas de estrógenos, Igualmente, según el Consenso Colombiano sobre Menopausia y Climaterio, se estableció que en las mujeres postmenopáusicas con osteoporosis, el aporte de calcio debe oscilar en un rango de 1 a 1,5 gramos diarios, correspondiendo mínimo a $800 \mathrm{mg}$ de calcio dietario. Para la dosis restante se pueden utilizar suplementos de calcio.

La suplementación diaria prolongada de 1 gramo de calcio elemental (carbonato de calcio) y $14 \mathrm{mg}$ de vitamina D, incrementa la absorción intestinal de calcio y por consiguiente la densidad mineral ósea lumbar en mujeres postmenopáusicas.

Se cree que los suplementos pueden aumentar el riesgo de formación de cálculos de oxalato de calcio. Por lo tanto, personas con historia de cálculos, hipertiroidismo primario e hipercalciuria renal, no deberían tomar suplementos.

El calcio existe en la naturaleza unido a otras sustancias a lo cual se le denomina compuesto. Los compuestos más comunes usados como suplementos incluyen carbonato, citrato, fosfato, gluconato y lactato de calcio. Cada uno contiene cantidades diferentes de calcio elemental, el cual es la cantidad real de calcio en el suplemento.

Carbonato de calcio ................... $40 \%$
Fosfato de calcio tribásico ............. $38 \%$
Citrato de calcio .......................... 27\%
Lactato de calcio. ......................... 13\%
Gluconato de calcio....................... 9\%

La mayoría de los productos en el mercado se absorben fácilmente en el organismo. Pero, son los compuestos a partir de carbonato los que se absorben mejor cuando se consumen simultáneamente con las comidas, mientras que las sales con base de citrato tie- 
nen buena absorción en cualquier momento del día. El citrato se absorbe mejor sin las comidas, pero en general la absorción de ambas formas es similar.

En general, los compuestos disponibles en el mercado son bien tolerados y con mínimo efectos adversos, siendo los más comunes el estreñimiento y la flatulencia. Esto se puede resolver casi siempre con una buena ingesta de líquidos y dieta rica en fibra. Las ingestas de calcio hasta $2.000 \mathrm{mg}$ día parecen ser seguras, ya que lo que sobre se excreta por la orina o la materia fecal. Pero, pueden ocurrir potenciales efectos adversos como resultado de altas ingestas por periodos prolongados.

En cuanto a interacción, la más importante es la alteración en la absorción de los suplementos de hierro, por lo cual se recomienda no ingerirlos concomitantemente. El calcio también interfiere con la absorción de la tetraciclina.

Así, aquellos individuos que por algún motivo no alcanzan a ingerir sus requerimientos diarios de calcio a través de la dieta, pueden obtener beneficio de algún suplemento de calcio. Sin embargo, es importante tener en cuenta cuál es la preparación que mejor se ajusta a las necesidades de cada paciente en forma individual. Como norma, debe preferirse un compuesto que contenga una alta concentración de calcio elemental por peso, se desintegre y absorba fácilmente en el tracto digestivo, que provea una forma de calcio que sea biodisponible, que sea barato, fabricado por una casa reconocida y que esté libre de tóxicos.

\section{Recomendaciones}

- Promover una cultura alimenticia adecuada, no solo para garantizar el cumplimiento de los requerimientos básicos de calcio, sino para mantener una buena masa ósea siempre, y que las pérdidas sean mínimas. El ejercicio, desafortunadamente muy restringido en la época actual, y otros buenos hábitos de vida son igualmente importantes.

- La educación es el elemento clave cuando se habla de prevención de la osteoporosis.

- Involucrar a otros sectores, como el de Educación (maestros, educandos, rectores), a los pediatras, a los padres de familia, en el proceso educativo y preventivo.

- Anamnesis nutricional.

\section{Estrógenos}

En estudios observacionales la terapia estrogénica posmenopáusica prolongada, con una adecuada suplementación de calcio, se asocia con una reducción del $50 \%$ a $60 \%$ en el riesgo de fracturas de cadera relacionadas con osteoporosis y de aproximadamente el $80 \%$ en el de fracturas vertebrales. Aumentan densidad mineral ósea en el hueso trabecular de columna lumbar en un $6 \%$ y en cadera y radio entre 2 y $3 \%$.

Sobre el riesgo de fracturas no existe ningún estudio clínico controlado que evidencien su efectividad. En un meta análisis se encontró un RR de 0.73 con mayor protección en menores de 60 años.

No han demostrado reducir la incidencia de nuevas fracturas vertebrales en mujeres mayores de 65 años, por esta razón en mujeres que tienen fracturas la terapia estrogénica sola no esta indicada como tratamiento, sino que debe estar combinada con otro tipo de fármacos.

No existe ninguna diferencia significativa respecto al tipo de estrógeno o vía de administración, sin embargo no hay evidencia tipo 1 con los estrógenos sintéticos.

1. Dosis: para mantener la masa ósea, son útiles las siguientes equivalencias de dosis para tratar a las mujeres posmenopáusicas sintomáticas: 17 beta estradiol 1 a $2 \mathrm{mg}$ / día, etinil estradiol 5 a 10 microgramos/ día, estrógenos equinos conjugados $0,625 \mathrm{mg} /$ día, estradiol micronizado ( parches) 50 microgramos/día y valerato de estradiol $2 \mathrm{mg} /$ día.

La terapia estrogénica sola tiene un RR de 5 para cáncer de endometrio, si se asocia gestageno se disminuye a 0,9 .

Se debe prescribir terapia estrogénica de manera continua, asociado a gestágeno en el caso de mujer con útero intacto, para reducir el riesgo de hiperplasia y carcinoma endometrial. El progestágeno se puede administrar de manera cíclica: medroxiprogesterona 5mg, 
acetato de noretindrona $2.5 \mathrm{mg}$ o progesterona micronizada 2()() $\mathrm{mg}$ ( en la noche) desde el día I hasta el 12 o 14 del mes, o bien de manera continua: medroxiprogesterona 2,5 mg/día, acetato de noretindronal 1,25 mg/ día o progesterona micronizada $1(0)$ mg/día.

2. Indicaciones: están aprobados por FDA paua prevención de osteoporosis; son útiles en todas las mujeres a partir de yue se diagnosticyue un déficit estrogénico fisiológico o inducido (quirúrgico), a menos (yue tengan algunal contraindicación absoluta como: neoplasia activa dependiente de estrógeno (mama y endometrio), hepatopatías agudas, trastomos tromboflebíticos o tromboembólicos activos y enfermedades sistémicàs graves nocontroladas (hipertensión anterial, diabetes, etcétera).

A favor de esta terapia estín sus efectos adicionales de control de la sintomatología climatérica como: oleatdas de calor, attrofïa genital, uretritis, irritabilidad, depresión, diticulades de concentración y adelgazamiento de la piel, produciendo una mejoría en la calidad de vida de las mujeres en menopausia.

Nuncal es demasiado tarde para iniciar el tratamiento. Los estudios han confirmado yue las mujeres con osteopenia, mayores de 6.5 años, y las mujeres con osteoporosis establecida, aumentan su densidad mineral ósea con terapia estrogénica; pero aun falta por delinir yue efecto tiene este incremento sobre la incidencia de fracturats.

3. Duración del tratamiento: la terapia estrogénical para prevención y manejo de osteoporosis debe durar un tiempo mínimo de 5 años, y probablemente debe ser de por vida, en ausencia de efectos colatterales. La matsa ósea se pierde rápidamente al suspender la terapia y puede alcanzar los niveles previos dentro de los primeros cuattro años de suspendida.

4. Monitoreo de seguridad: se recomienda examen anual de seno y mamograma, además de monitoreo endometrial con ultrasonido vaginal si se encuentra en terapia hormonal combinada; si el espesor endometrial es mayor de $5 \mathrm{~mm}$, es indicación para biopsia endometrial.

5. Efectos colaterales: mastalgia, aumento de los triglicéridos, aumento en las concentraciones de colesterol en bilis con un aumento relativo de 2 a 3 veces del riesgo de litiasis (efecto menor con la vía transdermical ). La náusea y el vómito constituyen una reacción inicial al tratamiento con estrógeno oral en algunas mujeres, pero puede desalparecer con el tiempo y minimizarse al ser tomados con los alimentos o justo antes de acostarse. Pueden causar migraña intensal en algunas mujeres, también es posible yue reactiven o exacerben endometriosis y el dolorconcomitante.

Sangrado por deprivación en las mujeres con tratamiento de progestágeno cíclico: en las mujeres en terapia combinada continua pueden tener sangrado repentino durante 4 a 6 meses antes de volverse amenorréicas y es una de las principales razones de abandono de la terapia, por ello se debe informar a las pacientes a cerca de lo yue deben esperar, antes de inicialr el tratamiento.

Con respecto al cáncer de seno no se puede afirmar yue existe una relación calusa efecto demostrada. Es posible que exista un increment() muy bajo en el riesgo de desarrollar cáncer de seno en mujeres con factores de riesgo y yue se incremente únicamente en casos de terapia hormonal de reemplazo (THR) por tiempo prolongado. Es importante tener en cuenta que en calso de que apare\%ca un tumor, este es de mejor pronostico.

Los progestágenos pueden reducir el efecto hipolipemiante de los estrógenos un 5()$\%$, pueden inducir resistencia periféricala la insulina y pueden aumentar el riesgo de vasoespasmo, dichos efectos se observan mas con medroxiprogesterona yue con progesterona micronizalda.

\section{Moduladores selectivos de los receptores de estrógenos}

1. Mecanismo de acción: modulan de manera selectiva los receptores de estrógenos. Ios activan pero tienen efectos diferentes en los tejidos a los del estradiol. teniendo efecto agonista sobre el sistema cardiovascular y el hueso y antagonista en el seno y a veces en el útero. A este grupo pertence el tamoxifeno, clomifeno, raloxifeno y la tibolona. 
El tamoxifeno en pacientes premenopáusicas disminuye densidad mineral ósea, no así en las mujeres con déficit estrogénico establecido, en quienes ha demostrado un incremento de densidad mineral óseal del ().8 al $1.4 \%$

El raloxifeno tiene un efecto similar al de los estrógenos sobre los marcadores de recimbio óseo y el calcio urinario, sin embargo con raloxifeno se incrementa la DMO en todos los sitios de manera global y sostenida en un 2-3\% y reduce el riesgo de fractura vertebral en un 4()-60 \%. Disminuye aparición de nuevas fracturas y reduce las fracturas múltiples en pacientes con osteoporosis, sin fracturas preexistentes. El raloxifenono aumenta el grosor endometrial y tiene efecto antagonistat en seno. Disminuye colesterol total (.3 a $6 \%$ ). LDL ( 4 a $1(0 \%)$, atumenta HDL.2 y efecto neutro sobre triglicéridos. Aumenta oleadas de calor.

2. Indicaciones: el raloxifeno esta aprobado por FDA para prevención y manejo de osteoporosis, especialmente útil en mujeres que no deseen la terapia hormonal de reemplazo o lat tengancontraindicada: pacientes con miomatosis uterina o endometriosis. Se deben iniciar con menopausia establecida, sin síntomas climatéricos.

Están contraindicados: en pacientes con trombosis venosia o trombofilia, alteración hepática, incluyendo colestasis y alteración renal severa.

3. Dosis: el raloxifeno se indica vía oral a dosis de 6() mg/día. con o sin alimentos, de manera continua. No requiere ajuste de dosis en pacientes de edad avanzada, se recomienda administrar suplementos de calcio. No se debe administral simultáneamente con colestiramina pues este fármaco reduce la absorción y el ciclo enterohepático del raloxileno

4. Duración del trat amiento: con raloxifeno hay estudios de cinco años de duración manteniendo su electo antirresontivo, pero se desconocen sus efectos a largoplazo.

5. Efectos colaterales: en los estudios clínicos con el medicamento se hat reportado una incidencia de $0,8 \%$ de episodios tromboembólicos venosos; bochornos en el 23,4\%, más frecuentes en los prime- ros seis meses de tratamiento; calambres en las piernas en el $5.5 \%$ y edema periférico en el $3,1 \%$.

\section{Bisfosfonatos}

Son compuestos análogos de los pirofostatos inorgánicos con una estructura P-C-P que se caracteriza por la presencia de 2 grupos fosfonato unidos a un soloátomo decarbono terminal. que los protege de las pirofosfatasas, y les conliere aviclezpor los cristales de hidroxiapatita inhibiendo la disolución del cristal. Seclasifican en tres generaciones, variando su potencia antirresontiva de menor a mayor. Primera generación: etidronalo, segunda generación: alendronato y tercera generación: risedronato.

Cada bisfosfonato liene sus propials característicats biológicass, farmacocinéticas y biocuúmicas. Tienen una biodisponibilidad baja. pues su absorción después de una dosis oral es baja ( 1 a $5 \%$ ) y no se metabolizan en forma sistémical, con un riesgo de interacción medicamentosa mínima. Se elimina la mitad de la dosis por orina al cabo de 24 horas y el $8.5 \%$ en orina en el curso de 28 días. El medicamento no absorbido es eliminado sin cambio por las heces. Alrededor del 30\% al $40 \%$ de la dosis absorbida, se adhiere a las superficies activas del hueso, se liberan lentamente del esqueleto, donde tienen una vida media prolongada y pueden durar toda la vida. No existen estudios en poblaciones menores de 18 años.

1. Mecanismo de acción: en los tejidos inhibe la resorción ósea al disminuir el numero y la actividad de los osteoclastos. En la célula inhibe el reclutamiento y adhesión de los osteoclastos, disminuye su vida media al incrementar su apoptosis debido a la inhibición de la vía del mevalonato intracelular y en su membrana.

Aumentan la densidad mineral ósea de 4-9\% en columna y de 1.6-6\% en cuello femoral. Alendronato y risedronato disminuyen el riesgo de fractura de columna y cadera y solo fractura de cadera con etidronato.

Un reciente meta análisis demuestra que el ctidronato reduce el riesgo de nuevas fracturas vertebrales en un $37 \%$ y en fracturats no vertebrales el 2()\%, en cadera en un .34\% y muñecal en un 19\%. Alendronato y risedronato 
reducen el riesgo de nuevas fracturas vertebrales aproximadamente en un $50 \%$ a tres años de tratamiento. Para facturas no vertebrales la reducción es de 30 a $40 \%$

2. Indicaciones: coadyuvante en el tratamiento y prevención de la osteoporosis posmenopáusica, senil, masculina e inducida por glucocorticoides. Alternativa efectiva de la THR para mujeres en quienes esta no sea atractiva, la tenga contraindicada o en mujeres en quienes persista la perdida ósea a pesar del uso de los estrógenos. Alendronato tiene dos estudios clínicos en uso combinado con THS, de uno y dos años de duración, que demostraron mayor incremento en la masa ósea y decrementos en el recambio óseo que cada una de las terapias por separado.

3. Dosis: para prevención: alendronato $5 \mathrm{mg} /$ día. Para tratamiento: etidronato $400 \mathrm{mg} /$ día por 14 días, en ciclos de cada 3 meses, alendronato $10 \mathrm{mg} /$ día o $70 \mathrm{mg}$ semanales y risedronato $5 \mathrm{mg}$ /día. El medicamento no debe masticarse o disolverse en la boca, debe administrarse con 6 a 8 onzas de agua pura, al levantarse, mínimo media hora antes del desayuno y mantenerse en posición erguida 30 minutos por lo menos, con el fin de evitar esofagitis, ulceras y erosiones del tracto digestivo alto. Se debe agregar calcio y vitamina $\mathrm{D}$, si la ingesta dietética es inadecuada, pero lejos de la administración del bisfosfonato, para evitar disminuir la absorción de este.

4. Duración del tratamiento: es variable y depende de la severidad de la pérdida de masa ósea y de la respuesta a la terapia. No se ha estudiado la seguridad del tratamiento por más de siete años.

5. Contraindicaciones: pacientes con estenosis o acalasia esofágica. Incapacidad para mantenerse en posición sentada erguida por lo menos 30 minutos, hipersensibilidad e hipocalcemia. Se recomienda usar con precaución en pacientes con antecedente de enfermedad ácido-péptica, el uso de antiácidos disminuye la absorción del medicamento.

6. Efectos colaterales: se han descrito dolor abdominal, nauseas, dispepsia, distensión abdominal, constipación, diarrea, dolor músculo-esquelético y rara vez exantema. En un estudio con alendronato se documen- tó la presencia de úlceras esofágicas en 3\% de los pacientes, por ello la insistencia en la adecuada toma del medicamento.

\section{Calcitonina}

Hormona polipeptídica producida por las células C parafoliculares de la tiroides, que se libera con niveles altos de calcio circulante, juega un papel importante en la homeostasis esquelética y mineral.

1. Mecanismo de acción: posee actividad antiosteoclástica, al unirse a receptores de dichas células disminuye su actividad resortiva y el reclutamiento de mas de ellas, con lo cual se inhibe la resorción ósea y la formación de nuevo hueso debido a un fenómeno de acoplamiento, que se observa durante el primer año de tratamiento. Incrementa la concentración de calcio y el AMPc en osteoblastos, incrementa el ARNm del colágeno tipo 1 y disminuye la fosfatasa ácida tartrato resistente.

2. Tipos de preparaciones disponibles: en la práctica clínica, la calcitonina más comúnmente utilizada se deriva del salmón y se consigue para administración parenteral: intramuscular o subcutánea e intranasal.

3. Efectos: a nivel histológico aumento de la masa ósea trabecular en un $13 \%$ disminución de la resorción en $32 \%$ y de la tasa de aposición en $10 \%$.

El estudio PROOF (evidencia 1) usó la calcitonina de salmón en spray nasal 200 UI día por cinco años y demostró: aumento de la DMO en columna lumbar de 1,0 a 1,5\%, la resorción fue inhibida (supresión de C telopéptido) en $12 \%$ y disminución de fracturas vertebrales nuevas en 33 a $36 \%$ en grupos con baja masa ósea o fractura vertebral preexistente. El máximo efecto se ha visto en pacientes osteoporóticos con resorción ósea incrementada en la columna más que en el esqueleto apendicular.

Efecto analgésico (evidencia 2) producido por inducción e incremento de beta endorfinas circulantes y efecto directo sobre los centros de umbral del dolor en el sistema nervioso central, que se observa a partir del segundo día de administración y lleva a movilización más temprana de estos pacientes 
4. Indicaciones: en 1985 la FDA aprobó la calcitonina para el manejo de la osteoporosis. Se recomienda en mujeres posmenopáusicas de edad avanzada, como alternativa de la terapia estrogénica y en caso de fracturas dolorosas. También esta recomendada en enfermedad de Paget e hipercalcemia.

5. Dosis: la dosis recomendada en aerosol nasal para el tratamiento de osteoporosis posmenopáusica es de 2()) UI diarias (equivalentes a 5() UI subcutáneas), altemando diariamente las fosas nasales, acompañadas de un aporte suficiente de calcio y vitamina D. Las formas inyectables se pueden usar post-fractura I()() UI. vía subcutánea durante 6 a 8 semanas, luego tres veces por semana durante los siguientes 6 meses y en mantenimiento 50 UI tres veces por semana. La dosis de 100 UI es inefectiva para prevenir la perdida ósea en la perimenopausia.

Se ha descrito resistencia a terapia continua a partir de 12 a 18 meses de tratamiento, por eso se recomienda administrarla en forma cíclica (tres meses sí, tres meses no).

6. Efectos secundarios: dosis dependiente, en I() a $20 \%$ de los pacientes en quien se administra de manera parenteral se puede presentar nausea, vomito, dolor abdominal, constipación, diarrea, rubor facial, prurito y adormecimiento de manos. Se puede presentar inflamación local en el sitio de inyección, efectos mas frecuentes en uso subcutáneo. Con el spray nasal se han descrito: rinitis y epistaxis en I () a $20 \%$ de los pacientes. No hay descritas interacciones.

7. Contraindicaciones: hipersensibilidad al compuesto. Existe recomendación de test cutáneo.

\section{Alfacalcidol y calcitriol}

Son metabolitos activos de la vitamina $\mathrm{D}$, aumentan la absorción intestinal de calcio y fósforo y la reabsorción renal del calcio, su producción se encuentra regulada por las necesidades de calcio en el organismo, en caso dehipocalcemia la glándula paratiroides libera PTH la cual activa la I-hidroxilasa renal que permite el paso de 25-hidroxicolecalciferol a calcitriol forma activa de la vitamina $\mathrm{D}$, que actúa sobre el osteoblasto en donde aumenta la síntesis del factor transformante de crecimiento beta y el numero de receptores del factor de crecimiento I, similar a la insulina, incrementan la síntesis de proteínas como la osteocalcina y osteopontina esenciales para la mineralización de las fibrillas colágenas en el hueso, siendo un factor esencial en el desarrollo de la masa pico en la adolescencia. En la tercera edad conservan el remodelado óseo, conservan la masa ósea al regular la actividad de la PTH y por lo tanto inhiben la resorción ósea en los diferentes tipos de osteoporosis. Regulan el crecimiento endocondral y a través de los receptores en los osteocitos, potencian la calidad ósea a través del mecanostato y podrían prevenir las fiacturas.

Tienen efecto sobre los músculos mejorando su función en especial su contracción, mejoran el dolor óseo y de partes blandas.

En osteoporosis senil esta bien documentado el hiperparatiroidismo secundario y la disminución del calcitriol como responsables de la fragilidad ósea y el incremento de la actividad osteoclástica, especialmente en mayores de 7() años, se disminuyen los receptores de vitamina $D$ en el intestino y en los huesos y la deficiencia estrogénica incrementa la disminución de la actividad de los receptores. Al igual en osteoporosis tipo I se observo una disminución en la actividad de la I hidroxilasa, donde los estrógenos son un importante cofactor, lo que lleva a un aumento de liberación de calcio del hueso y una reducción en niveles de PTH.

Su uso inicial fue para manejo de osteodistrofia renal, hipoparatiroidismo posquirúrgico e idiopático, raquitismo dependiente de vitamina D y osteomalacia.

El alfacalcidol es un análogo sintético de calcitriol y se metaboliza a calcitriol por su 25 hidroxilación en hígado logrando su forma activa: puede producir hipercalcemia.

Desde 1980 se empezaron a investigar para manejo de osteoporosis en los países nórdicos con resultados variables, algunos mostraron un incremento en la masa ósea de 10 y $20 \%$, en otros no se observo cambios. la conclusión de dichos estudios es que el efecto del calcitriol es adecuado en pacientes en donde el defecto de vitamina $\mathrm{D}$ contribuye a la patofisiología de la 
osteoporosis y varia conforme el estado nutricional para el calcio y seria más efectivo en mujeres con una ingesta baja de calcio.

En osteoporosis I y II la deficiencia de vitamina D esta demostrada, pero el uso de sus metabolitos activos no han logrado demostrar en forma contundente ser efectivos per se en la prevención de la osteoporosis posmenopáusica. Alfacalcidol ha sido útil en mejorar masa ósea en pacientes japoneses, pero la base de su acción parece ser el déficit latente de vitamina D de esta población. Calcitriol en algunos estudios ha demostrado efecto benéfico en prevenir fracturas vertebrales.

Si se val a usar calcitriol se debe restringir el calcio dietario a 7()() a $8(0)$ mg/ día, monitorizall calcio urinario, manteniendo niveles menores de 3()() mg/ día y aumentar la ingesta diaria de agua

Dosis recomendada en osteoporosis de calcitriol es de $0.25 \mathrm{mg}$, dos veces al día, y de alfacalcidol $1 \mathrm{mg} /$ día. Se deben monitorizar los niveles de calciosérico y creatinina a las 4 semanas, 3 y 6 meses y después a intervalos de 6 meses.

\section{Otros tratamientos para la osteoporosis}

Parä el manejo de la osteoporosis existen. además de los citados anteriormente. otros medicamentos que en un momento dado y bajo determinadals circunstanciats pueden convertirse en una alternativa terapéutica adecuada. Dentro de estos estas medicamentos de reciente investigación como la tibolona, la hormona paratiroidea.

Tibolona: es un esteroide sintético con efectos estrogénicos, progestacionales y androgénicos gracias a la actividad de sus metabolitos y particularmente sobre el hueso su efecto terapéutico se debe al metabolito 3 alfa OH. La tibolona tiene efectos sobre el hueso y los productos del metabolismo óseo similares a los encontrados con los estrógenos. Mejora la densidad mineral ósea en columna y cuello femoral en mujeres postmenopáusicas y ayuda a prevenir la perdida ósea inducida por agonistals de GnRH. Su dosis es de 2,5 mgrs/día y se debe mantener durante el tiempo en que se desee mantener sus beneficios.
Hormona paratiroidea: la paratomona (PTH). sería en teoría el tratamiento ideal para el manejo de la osteoporosis. La PTH es una hormona producida por las glándulas paratiroides como un péptido de 84 aminoácidos y es el principal regulador del calcio; las células blanco están localizadas en el hueso y en el riñón y se libera en respuesta a niveles bajos slecalcio, también posiblemente seal secretada en el timo y el cerebro en pequeñas cantidades La PTH tiene efectos anabólicos sobre el hueso y primordialmente ayuda a la formación del hueso. Tiene doble función: en dosis bajas actúa como agente anabólico óseo y en al tas dosis es el principal agente estimulante de la resorción ósea. En humanos la forma terapréutical usadda ha sido la PTH recombinante humana (1-34), con buenos efectos terapéuticos aunque con el inconveniente de su vía de aplicación, parenteral, y su corta vida media lo que ha obligado al desarrollo de agentes análogos con una vida media más prolongada que permita mayor comodidad para su aplicación. Los tratatmientos con PTH han demostrado un alumento de la DMO en los cuerpos vertebrales en periodos de 6 a 24 meses de tratamiento, se logra el picomáximo de acción a los 12 meses. Su dosis usual de la PTH varia entre 2() y 40 microgramos diarios aplicados en forma subcutámea. Habrá cue tener en cuenta los costos.

\section{Tratamiento de la osteoporosis}

Lineamientos generales:

- En los pacientes en quienes se indical un tratamiento preventivo, se insiste en la necesidad de seguir las recomendaciones previas a la instauración del tratamiento, especialmente exámenes complementarios.

- Para los pacientes en quienes se indical un tratamiento, es indispensable partir del diagnóstico de osteoporosis. Hatsta el momento, con sus limitaciones, el patrón de oro del diagnóstico es la absorciometría de energía dual de rayos X (DEXA).

- Tener en cuenta cue por ser patología crónica, es frecuente la bajaladherencia al tratamiento a largo plazo. Además, la eficacia de los tratamientos disponibles actualmente en el mercado está entre el 3()$\%$ y 5()$\%$. 
- Se insiste en el tratamiento no farmacológico, como la nutrición, el ejercicio y la exposición al sol en todos los pacientes, incluyendo acpuellos con riesgo para fracturarse.

- Es determinante el análisis cuidadoso de las circunslancias particulares del paciente para olieceropcionesterapéuticas individualizadas. Ademáis, el paciente debe conocer claramente los principales efectos colaterales, las contraindicaciones y los costos del producto que se le va a prescribir.

\section{Tamizaje de la osteoporosis}

\section{¿Cuál es la relación entre la masa ósea y el riesgo de fractura?}

No se puede definir directamente la probabilidad de una fractura con una determinación de densidad ósea. Ya c que se debe tener en cuenta que la densidad ósea se comporta como una variable intermedia en el proceso que lleva a una fractura.

La medida de la densidad ósea se mide comientemente como un promedio de densidad de hueso mineral dentro una región escaneada. Dicha medida tiene el inconveniente de no reflejar los cambios en la micro arquitectura ósea cyue ocurren durante el proceso de pérdida ósea; pero de todas maneras es hasta el momento la mejor manera de medir la fortaleza ósea.

La absorciometría de energía dual de rayos X (DXA) y la tomografía computarizada cuantitativa son las técnicas para la medida de la densidad mineral ósea. La manera aceptada de expresarla es a través de una estandarización de una distribución de frecuencias de medidas. Es decir se ha convenido expresarla en términos de unidades de desviación estándar (DE. ). De acuerdo con la OMS, los valores inferiores a 2,5 DE. respecto a valores promedios de personas jóvenes (también llamado valor T), definen la osteoporosis.

El riesgo relativo de liractura pélvica es de 2.6 veces (con un intervalo de confianza del 95\% de 2,0 a 3,5) por cada unidad negativa de desviación estándar a nivel de la pelvis. El riesgo relativo de fracturas vertebrales es de 2,3 (con un intervalo de contianza del $95 \%$ de 1,9 a 2,8 ) por calda unidad negativa de desviación estándar a nivel espinal. Para los demás sitios el riesgo relativo es de 1,5 veces (con un intervalo de confianza del $95 \%$ de 1,4 a 1,6) por cada unidad negativa de desviación estándar en el respectivo sitio.

Hay que tomar encuentacpue lass medidas de la región escaneada (por ejemplo cadera o vértebrass lumbares) predicen mejor las fracturas de esa misma región (fracturas de pelvis o vertebrales). Por otra parte, el valor predictivo de fracturas de las medidas de densidad ósea en pacientes individuales es pobre; $y$ que existe una importante sobreposición de valores de densidad ósea entre las pacientes fracturadas (con valores en rango no osteoporótico) y las pacientes que no presentan ningún tipo de fractura (con valores en rango osteoporótico).

\section{Tamizaje en masa}

Debemos recordar que una prueba de lamizaje debe tener una buena validez, en términos de ser altamente sensible, es decir cue tenga pocos falsos negativos, sino también de que el diagnóstico temprano, mejore drásticamente el pronóstico de la enfermedad de tal manera que redunde en una mejor calidad de vida en el largo plazo.

Si se toma en cuental cue el desenlace más importante en el diagnóstico precoz, prevención y tratamiento de la osteoporosis es la reducción en el numero de fracturas y cue la densitometría no clasifica adecuadamente el riesgo de presentar fractura No se recomienda el tamizaje en masal de grupos de población.

Esta afirmación se sustenta en el hecho de que cuando se toma como referencia una desviación estándar negativa (-1 DE) respecto al valor promedio para la edad, hasta un $70 \%$ de pacientes fracturadas podrían no ser identificadas como de alto riesgo a través de la densitometría (falsos negativos). Del 30\% restante (rotuladas de alto riesgo), tan solola mitad sufrirían fiacturas ( falsos positivos), De esta manera un porcentaje de pacientes recibiría un tratamiento de manera innecesaria. Si tomamos como referencia dos unidades negativas de desviación estándar (-2 DE), un 87\% de las fracturas podría no ser identificadas por la densitometría como de alto riesgo (falsos negativos). 
Otro aspecto que no favorecen la realización de tamizajes poblacionales es el hecho del bajo porcentaje de la población que atiende a los mismos (al menos en los países en donde se ha intentando implantar); el bajocumplimiento con el tratamiento a largo plazo y la baja eficacia de los tratamientos disponibles en la actualidad (30\% a 50\%).

Además, para que una intervención sea costo-efectiva, el costo del tamizaje y del tratamiento de un gran número de personas deberá ser sobrepasado por la reducción de los eventos y por un aumento en la calidad de vida en aquellas personas identificadas como de riesgo y a quienes daremos tratamiento. La falta de impacto de los datos disponibles en la sobre costos y costo-efectividad no permiten la identificación de una apropiada estrategia de tamizaje.

Por lo tanto, se aconseja realizar un tamizaje selectivo basado en la presencia de otros factores de riesgo asociados como pueden ser la edad mayor a 65 años, el peso bajo sin que sea validado el valor de estos estimadores de riesgo en nuestra población. Por otra parte el tamizaje estará indicado cuando el resultado influirá en la decisión de prescribir un tratamiento o aceptarlo por parte de la paciente, así cuando existe incertidumbre tanto en el médico como en la paciente acerca de tomar terapia de suplencia hormonal podría estar indicado. De esta manera parecería innecesario realizarla en una mujer quien ha decidido tomar estrógenos por cualquier otra razón.

\section{¿Cuándo repetir el examen?}

De acuerdo a las categorías diagnósticas basadas en umbrales de desviación estándar establecidas por Organización Mundial de la Salud, se propone:

\begin{tabular}{lll}
$\begin{array}{l}\text { Desviaciones } \\
\text { estándar }\end{array}$ & $\begin{array}{l}\text { Tiempo para } \\
\text { repetir el examen }\end{array}$ & Tratamiento \\
Mayor a +1.0 & 5 a 10 años & No \\
\hline 0 a +1 & 5 a 10 años & No \\
\hline-1 a 0 & 2 a 5 años & No \\
\hline-1 a -2.5 & I año & $\begin{array}{l}\text { Considerar } \\
\text { tratamiento } \\
\text { preventivo }\end{array}$ \\
\hline Menor a -2.5 & 6 meses a I año & $\begin{array}{l}\text { Fuertemente } \\
\text { recomendado }\end{array}$
\end{tabular}

Recomendaciones del jurado

El estado deberá establecer políticas educativas dirigidas a padres de familia, médicos generales, pediatras y ginecólogos, instituciones educativas y a la comunidad en general que promuevan acciones de prevención de la osteoporosis que incluyan actividad física regular enfatizando en ejercicio de carga de peso y la dieta rica en calcio.

El uso sistemático de la historia clínica dirigida según la edad, para la detección de factores de riesgo de baja masa muscular; de riesgo de caída; riesgo de fractura del fémur o de las vértebras y factores de riesgo de osteoporosis ya sean modificables tales como: cigarri1lo, peso bajo, dieta baja en calcio, uso de estrógenos o no modificables como la desnutrición.

Validar las escalas de detección de riesgo de osteoporosis, en la población colombiana.

El manejo del paciente con osteoporosis debe ser considerado dentro de un programa de rehabilitación integral y multidisciplinario. También se debe incluir específicamente el programa de rehabilitación post fracturas.

El estado a través de las instituciones competentes deberá fijar las políticas para la prevención, el tamizaje y el tratamiento de la osteoporosis, dentro de la legislación de seguridad social vigente y debe también considerar los resultados de este consenso.

El estado deberá favorecer la evaluación del desempeño en nuestro medio del as tecnologías utilizadas en le diagnóstico y tratamiento de la osteoporosis. Así mismo deberá crear mecanismos tendientes a controlar el uso de las pruebas aplicadas hasta que se demuestre su efectividad, exactitud o eficiencia. 